\title{
Evaluación de la incorporación de un foro virtual por redes sociales entre estudiantes de odontologia chilenos
}

\author{
Assessment of the incorporation of a virtual forum through \\ social media among Chilean dental students
}

\author{
Debbie-J Álvarez-Cruces ${ }^{1}$; Maite Otondo-Briceño²; Alejandra del P Medina-Moreno ${ }^{1}$
}

Forma de citar: Álvarez-Cruces DJ, Otondo-Briceño M, Medina-Moreno AP. Evaluación de la incorporación de un foro virtual por redes sociales entre estudiantes de odontología chilenos. Rev Univ Ind Santander Salud. 2019; 51(2): 117-128. doi: http://dx.doi.org/10.18273/revsal.v51n2-2019004 @) (1)

\section{Resumen}

Introducción: Las redes sociales son cada vez más usadas en la enseñanza debido a su facilidad de uso, familiarización, accequibilidad, siendo posible el intercambio de información de manera instantánea y fácil desde teléfonos inteligentes. Por ello se diseñó y creó un foro virtual mediante un grupo cerrado de Facebook que funcionó de manera paralela a la asignatura. Objetivo: Evaluar la opinión de los estudiantes en relación al desarrollo de las habilidades actitudinales, cognitivas, autorreguladoras y metacognitivas mediante el foro virtual implementado, además de su percepción con respecto a la plataforma de Facebook. Metodología: Estudio transversal descriptivo de tipo mixto. Cuantitativo con la aplicación de una encuesta de opinión tipo Likert creada por Andrei Fedorov (2008) y cualitativo mediante cuatro grupos focales. Participaron 68 estudiantes ( 28 hombres y 40 mujeres), que cursaron la asignatura Diagnóstico Integrado el año 2017, correspondiendo al 100\% de ellos. Resultados: Para el $70 \%$ de las preguntas la opción más seleccionada fue -bastante-; a excepción de cuatro de la dimensión actitudinal donde la más frecuente fue -un poco-. Destacaron la plataforma de Facebook como una excelente idea para incentivar el aprendizaje, ofreciendo más ventajas que otras plataformas. Las desventajas mencionadas surgieron de los estudiantes con menor participación y regular/bajo rendimiento. Conclusión: El foro virtual, por medio de Facebook, permitió mejorar las dimensiones actitudinal, cognitiva, autorreguladora y metacognitiva, principalmente en mujeres y en aquellos que participaron más, obteniendo mejor rendimiento. Resultó ser una mejor plataforma virtual dada que es amigable, masiva e instantánea incentivando la motivación del estudiante.

Palabras clave: Foro virtual; Redes sociales; Educación en odontología; Habilidades actitudinales; Habilidades cognitivas; Habilidades autorreguladoras y metacognitivas.

1. Universidad de Concepción, Chile.

2. Universidad Católica de la Santísima Concepción, Chile.

Correspondencia: Debbie Jeinnisse Álvarez-Cruces. Dirección: Roosevelt 1550, Concepción, Chile. Teléfonos: +56 412204291. Correo electrónico: debbiejalvarez@udec.cl. 


\begin{abstract}
Introduction: Social networks are increasingly used in education due to its ease of use, familiarization, accessibility, making it possible to exchange information instantly and easily from smartphones. For this reason, a virtual forum was designed and created through a closed group of Facebook that worked in parallel with the subject. Objective: To evaluate the opinion of students in relation to the development of attitudinal, cognitive, self-regulatory and metacognitive skills through the virtual forum implemented, as well as their perception regarding the Facebook platform. Methodology: Cross-sectional descriptive study of mixed type. Quantitative with the application of a Likert opinion survey created by Andrei Fedorov (2008) and qualitative through four focus groups. 68 students participated (28 men and 40 women), who took the Integrated Diagnostic course in 2017, corresponding to $100 \%$ of them. Results: For $70 \%$ of the questions, the most selected option was -quite-; except for four of the attitudinal dimension where the most frequent was -a little-. They highlighted the Facebook platform as an excellent idea to encourage learning, offering more advantages than other platforms. The mentioned disadvantages arose from students with less participation and regular/ low performance. Conclusion: The virtual forum, through Facebook, allowed to improve the attitudinal, cognitive, self-regulating and metacognitive dimensions, mainly in women and in those who participated more, obtaining better performance. It turned out to be a better virtual platform given that it is friendly, massive and instantaneous which encourages student motivation.
\end{abstract}

Keywords: Virtual forum; Social networks; Dental education; Attitudinal skills; Cognitive skills; Self-regulator and metacognitive skills.

\section{Introducción}

Las Tecnologías de la Información y Comunicación (TIC) ya están instaladas en nuestras aulas universitarias. $\mathrm{Su}$ origen se remonta desde el momento en que el desarrollo electrónico, informático y tecnológico irrumpen de manera drástica a mediados del siglo $\mathrm{XX}^{1}$. En sus inicios, si bien complementaban las materias o contenidos, mantenían un patrón tradicional donde se transmitía información, como lo fueron las grabaciones de voz o los videos creados por los mismos profesores ${ }^{2}$.

Lo que marca una diferencia sustancial, a finales de los años 90, es la creación e incorporación de internet, permitiendo la interacción de estudiantes con profesores y entre estudiantes mediante los correos electrónicos y plataformas institucionales en el proceso formativo ${ }^{3,4}$. Es así que, hoy en día, ya no es tan necesaria la creación de plataformas virtuales institucionales para interactuar con los estudiantes, las redes sociales han venido a sustituirlas con características y cualidades insuperables como por ejemplo que: poseen múltiples y variadas funciones, son fáciles de utilizar, todos están familiarizados con ellas y, actualmente, es posible conectarse gracias a los planes de navegación que incluye redes sociales gratis o mediante una red inalámbrica abierta, más conocida como wifis. A ello se suma, las mayores posibilidades de adquirir teléfonos inteligentes que permite el intercambio de información de manera instantánea y fácil, sin tener que estar frente a un computador, accediendo mediante claves como tampoco adaptarse a las diferentes plataformas virtuales existentes ${ }^{1,4,6}$.
En un estudio The Economist Intelligence Unit y Facebook, donde se midió el índice de internet inclusivo en el año 2018, que analizó a 86 países considerando 54 indicadores en cuatro categorías (disponibilidad, asequibilidad, relevancia y preparación), Chile subió al puesto número ocho a nivel mundial y es el primer país latinoamericano, seguido por Brasil con el lugar 28, México 29, Argentina 34, Perú 38 y Colombia 44,8. Ello evidencia el enorme potencial que implica utilizar estos medios para la producción del conocimiento y acceso a la información, posibilitando una amplia variedad de estrategias que permiten motivar al estudiante.

Actualmente, son múltiples los estudios que avalan el uso de las TIC en la Educación Superior, no sólo porque a los estudiantes les sea atractivo y familiar, sino porque les fomenta un rol más activo en su proceso formativo. Esto se traduce en mejores capacidades actitudinales, permitiéndoles desarrollar, entre otras cualidades, las estrategias cognitivas, autorregulación y metacognición ${ }^{9-11}$. Al respecto Castro, et al. ${ }^{12}$ refieren que el foro virtual "tiene una dimensión cognitiva en la que se conjugan las habilidades intelectuales de alto nivel: análisis, inferencia, interpretación, explicación, evaluación y autorregulación, mismas que sustentan el pensamiento de calidad, caracterizado por ser lógico, racional, claro, transparente, sintético, reflexivo, contextualizado, oportuno, argumentado y autorregulado. Estos aspectos toman formas de operaciones intelectuales, comunicativas, metacognitivas y autorregulativas". 
Por su parte, el profesor asume un rol más pasivo pero, a la vez, cumple un papel fundamental e indispensable, puesto que debe dedicar gran esfuerzo y tiempo en todo el proceso ${ }^{1,3,9}$. De este último depende una buena planificación, metodología, creación y organización de la herramienta, puesta en marcha, monitorización del proceso y la retroalimentación que irá nutriendo al estudiante, para que el proceso de enseñanzaaprendizaje resulte exitoso $0^{1,3,9-11,13,14}$.

La asignatura de Diagnóstico Integrado de la carrera de Odontología de la Universidad de Concepción (UDEC), tiene como objetivo principal preparar al estudiante para que inicie sus actividades de atención clínica con paciente el siguiente año. Posee un programa extenso que incluye, entre otras, las asignaturas de promoción y prevención de la salud, semiología, patología general, embarazo, técnicas odontológicas básicas, nomenclatura odontológica y patología bucal prevalente, además de retomar los contenidos de ciencias básicas con el propósito de integrarlos para que el estudiante logre interrelacionar todos los factores que se ven involucrados al momento de diagnosticar y planificar el tratamiento del paciente. Existen seminarios conformados por 20 alumnos, donde se trabaja con la metodología de Análisis de Caso Clínico (ACC), además de actividades de práctica clínica en la cual se realizan procedimientos y técnicas de manera progresiva que le permitirán, al estudiante, adiestrarse en habilidades y técnicas propias de la profesión. Estas dos últimas metodologías son importantes y significativas para el alumno, puesto que pone en práctica lo revisado teóricamente en clases.

No obstante, en un estudio previo de las mismas autoras $^{15}$, los estudiantes evidenciaron conocer las estrategias cognitivas y metacognitivas necesarias para lograr un mejor aprendizaje; a pesar de ello, no las ejecutaban o no lograban seleccionarlas y/o adaptarlas a las diferentes situaciones. Lo que se condice con otros estudios similares ${ }^{6}$. Además, señalaron la necesidad de instalar más oportunidades para integrar y aplicar lo que aprendían, ya que, según ellos, no sólo les permitiría un mejor desempeño en la asignatura, sino mayor seguridad en el quehacer de práctica clínica del siguiente año de estudio. Por lo mismo, dado los pobres resultados obtenidos en el desarrollo de las estrategias cognitivas y metacognitivas en el grupo descrito, como a la necesidad patente de los estudiantes, apremiaba realizar una intervención educativa que contribuyera a mejorar las competencias que los preparara de mejor manera para la atención de pacientes.

Dado lo anterior, se diseñó y creó un foro virtual que funcionó de manera paralela a la asignatura. Este debió cumplir con ciertos requisitos y características, como: ser accesible, de fácil uso, de participación libre y voluntaria. En él, se abordaron los diferentes contenidos de la asignatura con la metodología de ACC, que les permitiera la contextualización de problemas, favoreciera la integración de los contenidos, les ayudara a reflexionar y pudieran extrapolar dichas situaciones a su futuro próximo como profesionales. La plataforma virtual seleccionada fue un grupo cerrado de Facebook, ya que cumplía con las características mencionadas.

Cada vez es más frecuente observar la utilización de las redes sociales en el proceso de enseñanza-aprendizaje, sin embargo son escasos los estudios que se han llevado a cabo para evaluar si contribuyen de manera positiva o no. Hasta el momento se han limitado a cuantificar la plataforma más utilizada, determinar para qué la utilizan, estratificar a los participantes por grupo etáreo y realizar revisiones bibliográficas ${ }^{4,14,16}$.

Por todo lo anterior, resulta necesario evaluar la opinión de los estudiantes de la asignatura Diagnóstico Integrado de la carrera de Odontología de la UDEC en relación al desarrollo de las habilidades actitudinales, cognitivas, autorreguladoras y metacognitivas mediante el foro virtual implementado, además de su percepción con respecto a la plataforma de Facebook.

\section{Metodología}

Se realizó una investigación descriptiva de corte transversal. Se utilizó metodología mixta: cuantitativo y cualitativo.

\section{Enfoque cuantitativo}

Se escogió el instrumento creado y validado por Andrei Fedorov ${ }^{17}$ en el año 2008 (Anexo 1) ya que mide las variables de interés como son las habilidades actitudinales, cognitivas, autorreguladoras y metacognitivas. Este ya ha sido utilizado en Chile en otras investigaciones para la evaluación de foros ${ }^{12}$, por lo que no fue necesaria una nueva validación y adaptación.

El instrumento consta de 20 preguntas con una escala tipo Likert de 5 puntos, donde 1 corresponde a "para nada”, 2: “muy poco", 3: "un poco", 4: "bastante” y 5: "por completo”. Mide tres dimensiones, definidas así:

1) Actitudinal: incluye un amplio y complejo conjunto de actitudes y disposiciones mentales necesarias para mantenerse bien informado, investigar $\mathrm{y}$ 
aprender, generar la opinión propia, evidenciar el sentido crítico y negociar las ideas en forma inteligente y oportuna. Incluye las preguntas $\mathrm{N}^{\circ} 2$, 7, 11, 13, 14, 15, 16, 17, 18 y 20.

2) Cognitiva: capacidad cognitiva de alto nivel o habilidad para comprender, analizar, reflexionar, depurar, sintetizar y argumentar las ideas complejas; elaborar un juicio de valor acerca las ideas propias y de los demás, que se confrontan en medio de una búsqueda de un consenso inteligente. Incluye las preguntas $\mathrm{N}^{\circ} 1,3,4,9$ y 10 .

3) Autorregulativa y metacognitiva: cualidades que procuran la ética y la calidad del pensamiento crítico. Por ejemplo: la consideración del contexto, conciencia de repensar las ideas, de buscar la claridad de expresión y la oportunidad de uso del pensamiento crítico y respeto por la opinión crítica e inteligente de los demás. Incluye las preguntas $\mathrm{N}^{\circ}$ $5,6,8,12$ y 19 .

La muestra fue no probabilística por conveniencia y estuvo constituida por un universo de 68 estudiantes (28 de sexo masculino y 40 del femenino) que cursaron la asignatura de Diagnóstico Integrado de la carrera de Odontología el año 2017, lo cual correspondió al $100 \%$ de los estudiantes. Además de aceptar participar voluntariamente por escrito mediante consentimiento informado.

\section{Enfoque cualitativo}

Se realizó mediante cuatro grupos focales conformados por seis estudiantes en cada uno de ellos (15 sexo femenino y 9 del masculino). El muestreo fue de tipo aleatorio simple dentro del universo descrito anteriormente (68 estudiantes). El guión temático del focus group (Anexo 2) tuvo como propósito conocer la percepción de los estudiantes en relación a la utilización de la plataforma de Facebook para un foro virtual en la asignatura Diagnóstico Integrado de la carrera de Odontología de la UDEC. Tuvo una categoría de análisis principal denominada -Plataforma de Facebook-, con tres subcategorías: Ventajas, Desventajas y Sugerencias del foro.

\section{Procedimiento}

Se creó un grupo cerrado de Facebook donde se trabajó con ACC de diferente complejidad sobre los contenidos vistos en clases. El foro virtual estuvo activo entre los meses de marzo a julio del año 2017. Fue de libre participación. Participaron 2 docentes que, aparte de subir ACC a la plataforma dos veces a la semana como mínimo, debían retroalimentar a los estudiantes.

Una vez finalizado dicho tiempo, se procedió a realizar la encuesta de opinión de Fedorov y los focus group en un horario acordado con los estudiantes.

\section{Resultados}

\section{Resultados cuantitativos}

Se utilizó el programa SPSS versión 23, que permitió analizar los resultados de las variables de interés y establecer descripciones de ellas. En la Figura 1 se puede observar que los resultados son bastantes satisfactorios, ya que los porcentajes se concentran en la opción -bastante-, seguida de -un poco- Ello pone de manifiesto, que el foro virtual, tuvo un real impacto en los estudiantes para todas las preguntas que incluye la encuesta.

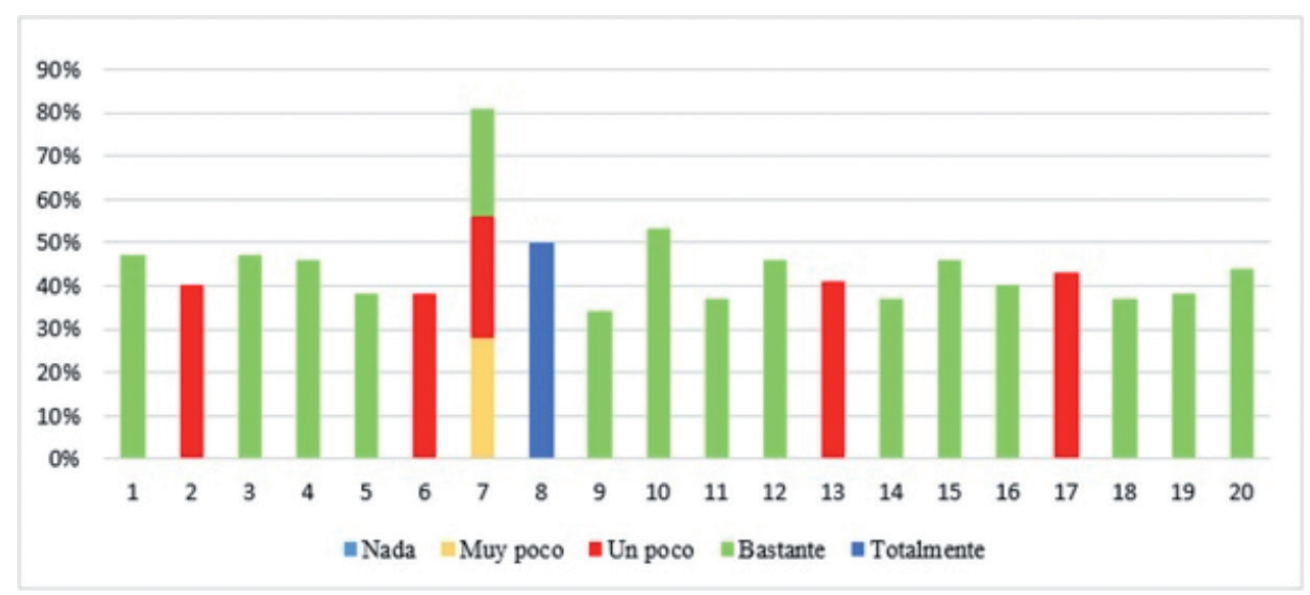

Figura 1. Porcentaje de respuestas más frecuentes para la encuesta de opinión de Fedorov. 
Se realizó agrupamiento de datos para las dimensiones actitudinal, cognitiva $\mathrm{y}$, autorregulativa y metacognición. En la Figura 2 se observa que, para la primera dimensión, las preguntas $\mathrm{N}^{\circ} 11,14,15$, 16, 18 y 20 la opción más frecuentemente dada por los estudiantes fue -bastante- seguida de -un poco- a excepción de la 20 que le sigue la opción -totalmente-. Para las 2, 7, 13 y 17, que se relacionan con la actitud, conducta y disposición para investigar y aprender, la preferencia se mantuvo en -un poco-

Para todas las aseveraciones que componen la dimensión cognitiva, la respuesta más frecuentemente seleccionada fue -bastante- Y, para la dimensión autorregulativa y metacognición, para la pregunta 8 fue -totalmente-, la 6 con -un poco- seguida de -bastante- y para el resto de preguntas que componen esta dimensión (5, 12 y 19), -bastante-

Ello pone en evidencia que, a visión de los estudiantes, el foro virtual permitió desarrollar las habilidades actitudinales, cognitiva, autorregulativa y metacognición. Aunque es importante señalar que, la actitud y disposición necesaria para buscar situaciones de aprendizaje se mantiene limitada, quizás se deba al mismo rol pasivo al cual se han acostumbrado adoptar ${ }^{15}$.

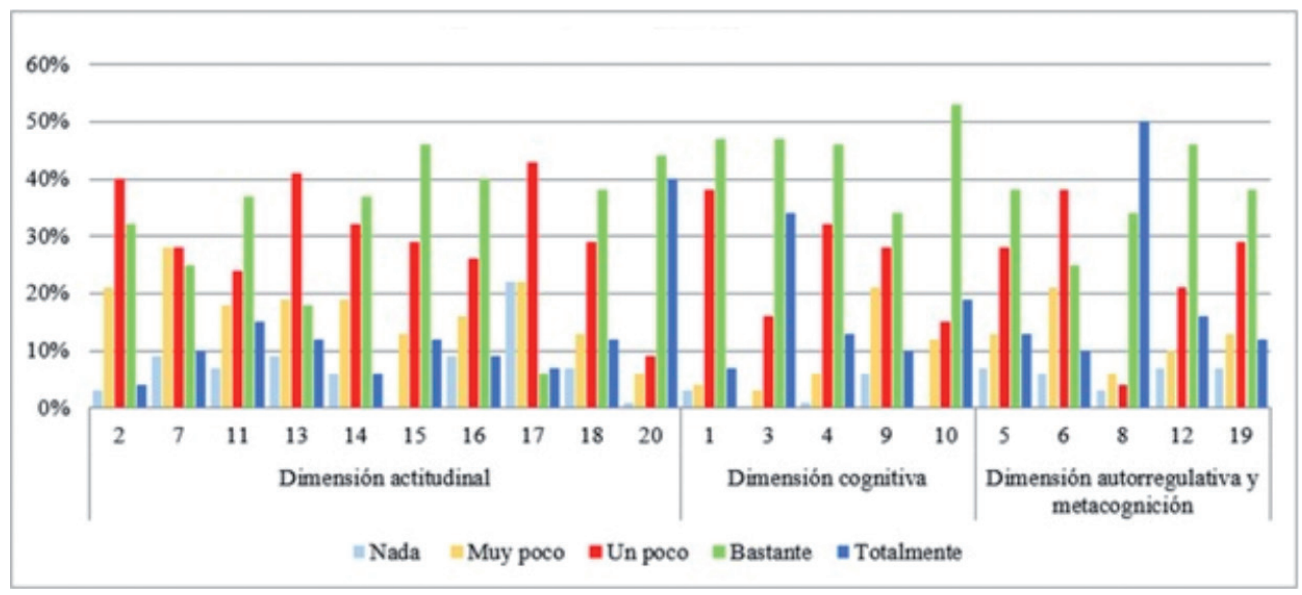

Figura 2. Porcentaje de respuestas agrupadas por dimensiones para la encuesta de opinión de Fedorov.

Se realizó triangulación de datos por género, participación en el foro y rendimiento académico obtenido en la asignatura. Los criterios de selección se estandarizaron de la siguiente manera:

- Género: femenino y masculino.

- Alta participación en el foro: para los estudiantes que hayan participado 5 o más veces durante el semestre académico.

- Baja participación en el foro: para quienes hayan participado 4 o menos veces durante el semestre académico.

- Alto rendimiento en la asignatura: promedio de notas obtenido por el estudiante mayor o igual a 5,7 (en una escala de 1 a 7).

- Regular o bajo rendimiento en la asignatura: promedio de notas obtenido por el estudiante menor o igual a 5,6 (en una escala de 1 a 7).

En la Figura 3 es posible observar la gráfica comparativa entre el género femenino y masculino. Las mujeres respondieron de manera más positiva todas las preguntas, dando cuenta de que la metodología utilizada les permitió mejorar la manera en que aprendían, por lo que el foro virtual desarrollado fue mejormente aprovechado por este grupo que por los de género masculino. La única excepción la constituye la pregunta 5: Siento que con el foro he sido estimulado a ser vigilante en las oportunidades de usar el pensamiento crítico, que fue mayor tanto para la opción -bastante- como -totalmente- para el género masculino. Lo anterior, podría estar dado porque la tecnología incorporada estimuló más a estos últimos que a sus contrapartes, evidenciando que los varones de la asignatura necesitan de una mayor motivación extrínseca para aprender.

En la Figura 4 podemos observar la comparación por participación en el foro. Para el grupo que tuvo una alta participación las respuestas son proporcionalmente más para las opciones -bastante- y -totalmente-; a diferencia del grupo que tuvo una baja participación, donde se destaca un menor porcentaje para dichas opciones, e incluso, es posible observar que, en varias preguntas, aparece la opción 3. La única excepción la constituye la pregunta 12: Creo 
que una de las consecuencias del foro es que ahora estoy más consciente de repensar mis opiniones antes de expresarlas, lo que se condice bastante con los que participaron poco, puesto que, al finalizar el proceso, se dieron cuenta que fue una herramienta valiosa para su aprendizaje a pesar de no haber participado tanto.

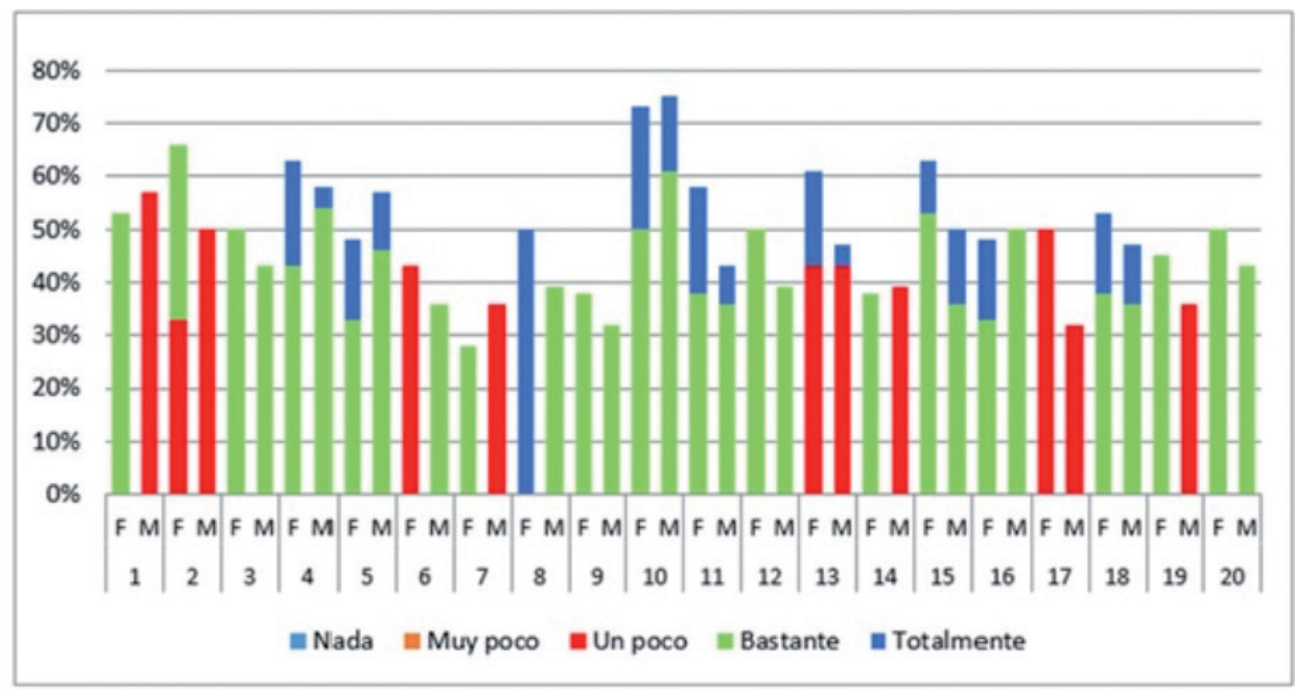

Nota: F: Femenino; M: Masculino.

Figura 3. Comparación de porcentajes de respuesta por género de la encuesta de opinión de Fedorov.

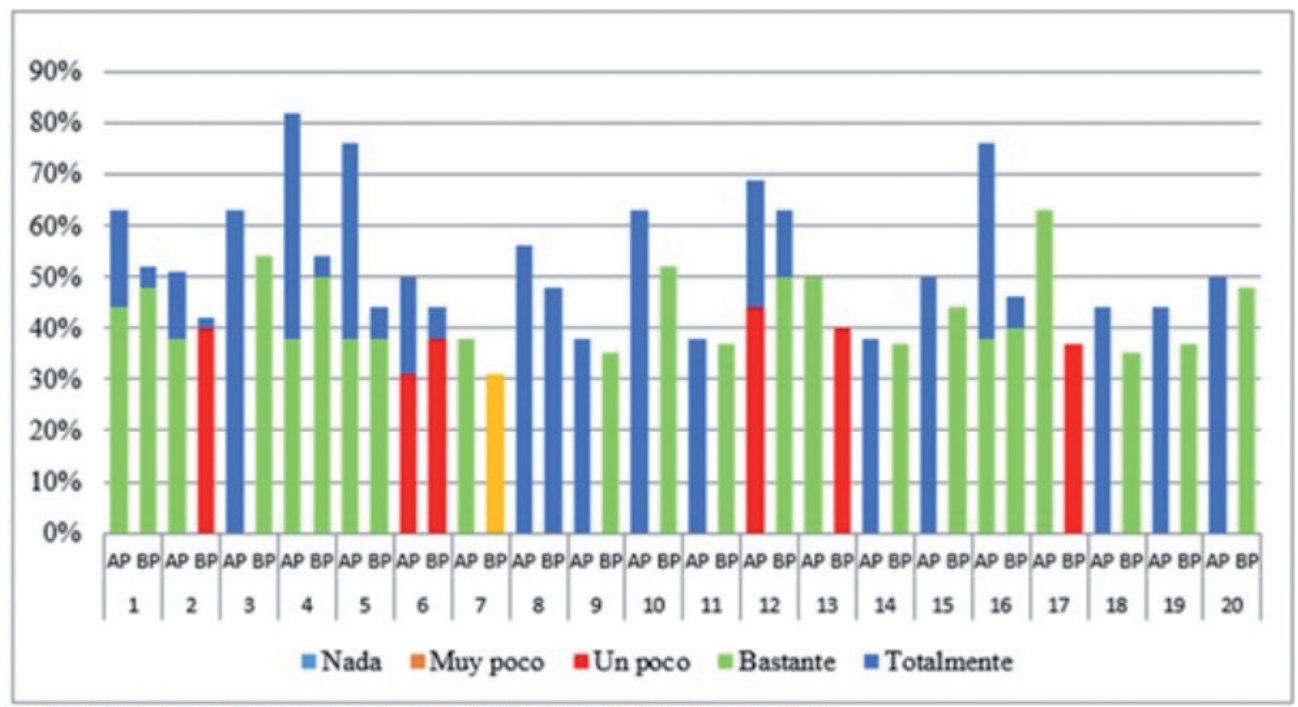

Nota:AP: Alta participación en el foro; BP: Baja participación en el foro.

Figura 4. Comparación de porcentajes por participación en el foro para la encuesta de opinión de Fedorov.

Finalmente, al comparar el alto rendimiento académico obtenido en la asignatura con el regular/ bajo rendimiento, es posible apreciar, en la Figura 5, que la diferencia es sustancial en favor de aquellos que tuvieron mejor rendimiento. De tal manera que, las respuestas se concentran en la opción -bastante- y -totalmente-; a excepción de las preguntas 5: Siento que con el foro he sido estimulado a ser vigilante en las oportunidades de usar el pensamiento crítico y 7 :
Siento que el espíritu investigativo ha sido impulsado por medio del foro, donde el grupo de menor rendimiento académico mostró mayor adherencia a las opciones -bastante- y -totalmente-, a diferencia del otro grupo que optó por la opción -un poco- y -mиy росо-. Ello podría estar dado porque, los de alto rendimiento, piensan que no necesitan de más acciones que fomenten sus habilidades, puesto que ya lo tienen como hábito; en cambio, los de rendimiento regular/ 
bajo, necesitan de herramientas o metodologías que incentiven su interés por el aprendizaje. Es así que, los que tuvieron una alta participación en el foro lograron un alto rendimiento en la asignatura.

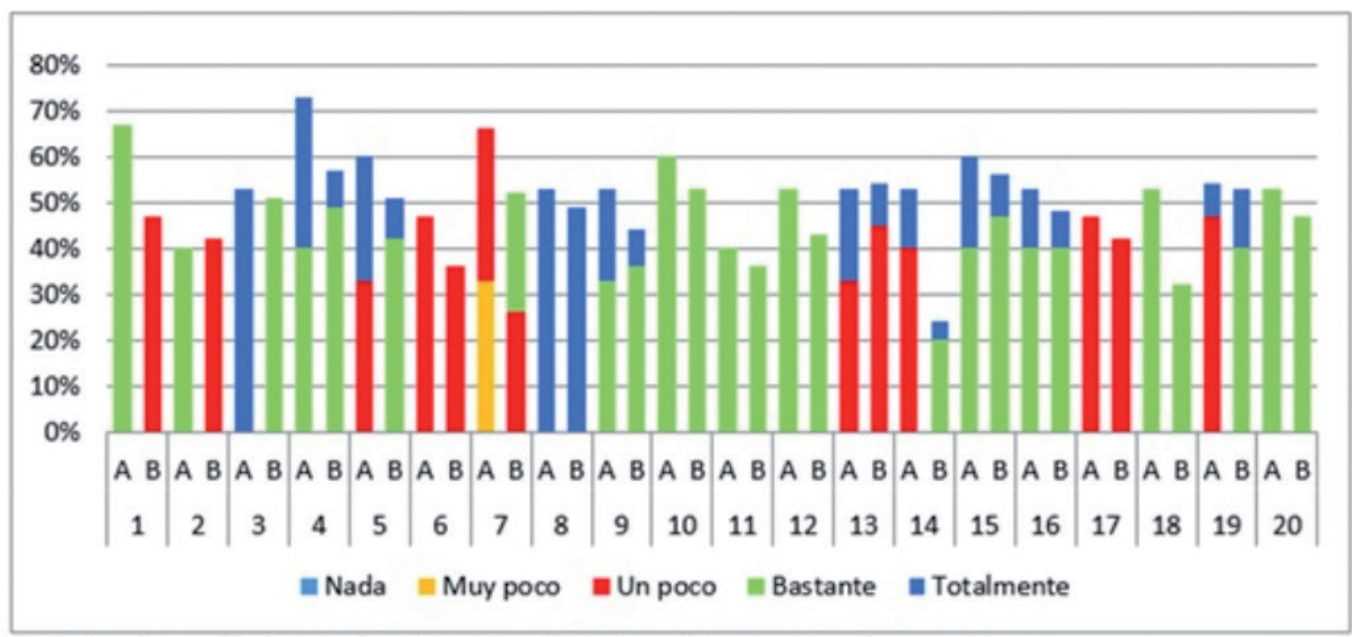

Nota: A: Alto rendimiento en la asignatura; B: Bajo rendimiento en la asignatura.

Figura 5. Comparación de porcentajes de respuesta por rendimiento académico de la encuesta de opinión de Fedorov.

\section{Resultados cualitativos}

Se transcribió las grabaciones de los cuatro grupos focales, el corpus textual fue analizado mediante el software NVivo versión 11. Se procedió a separar las citas en las subcategorías ya mencionadas, describirlas, asignarle una etiqueta y obtener la frecuencia de cada una de ellas. En total fueron 56 citas para las tres subcategorías.

\begin{tabular}{|c|c|c|c|}
\hline Subcat & Descripción & Etiq & Frec \\
\hline Ventajas & $\begin{array}{l}\text { Se refiere a las opiniones a favor } \\
\text { que los estudiantes refirieron en } \\
\text { relación al uso de la plataforma de } \\
\text { Facebook para el foro. }\end{array}$ & V & 31 \\
\hline Desventajas & $\begin{array}{l}\text { Se refiere a inconvenientes que } \\
\text { los estudiantes manifestaron en } \\
\text { relación al uso de la plataforma de } \\
\text { Facebook para el foro }\end{array}$ & $\mathrm{D}$ & 8 \\
\hline Sugerencias & $\begin{array}{l}\text { Mejoras mejora que los estudiantes } \\
\text { mencionaron para incorporar en } \\
\text { la plataforma de Facebook para el } \\
\text { foro }\end{array}$ & $\mathrm{S}$ & 17 \\
\hline \multicolumn{3}{|c|}{ TOTAL DE CITAS: } & 56 \\
\hline
\end{tabular}

Nota: Subcat: Subcategoría; Etiq: Etiqueta; Frec: Frecuencia.

Con el propósito de salvaguardar los criterios de calidad de los grupos focales, se desarrolló un procedimiento de triangulación de fuentes, es decir, se compararon las diferentes opiniones de los estudiantes según género, participación en el foro y los resultados académicos obtenidos en la asignatura, tal como se estandarizó en el análisis cuantitativo.

\section{Ventajas de utilizar la plataforma de Facebook para el foro virtual en comparación a otras}

A los estudiantes les pareció una excelente iniciativa el haber empleado un grupo cerrado de Facebook para motivar el aprendizaje, confesaron que, la mayor parte del tiempo, la utilizan para distraerse y evadir momentos de responsabilidad, por lo que es plausible la idea de haber aprovechado esta plataforma virtual para algo más productivo. Resaltaron el beneficio de utilizar esta plataforma en comparación a otras, como las institucionales (Arco e Infoda), refiriendo que son muy poco atractivas, de difícil operatividad, teniendo que orientarse y capacitarse para utilizarlas, y los limita a estar sólo en el computador puesto que en el teléfono celular no funcionan bien.

Otras ventajas mencionadas, para la plataforma de Facebook, tenemos que les resulta familiar, atractiva, muy accesible, rápida, la gran mayoría tienen acceso a ella, hay acceso a redes sociales gratis gracias a los nuevos planes de telefonía móvil, no es necesario que deban conectarse al computador, les avisaba de nuevas notificaciones generadas por el grupo, se podía responder inmediatamente a las preguntas que se generaban y si no, se podían ir leyendo las respuestas de los compañeros. De cualquier forma incentivaba el aprendizaje, ya que eran ACC relacionados con los 
contenidos revisados en la asignatura, por lo cual, no sólo les permitía observar la aplicabilidad práctica de la materia, sino que también les permitía reforzarla con las preguntas. Para los que no recordaban la materia, les incentivaba a revisar sus apuntes para responder de manera certera, otros no podían responder porque tenían otra clase en el mismo horario, pero después leían las respuestas de sus compañeros y les ayudaba para aprender y a otros, que antes utilizaban esta herramienta como distracción y ocio, les resultaba una utilización más productiva de su tiempo.

\section{Desventajas de utilizar la plataforma de Facebook}

Entre las desventajas tenemos principalmente cuatro:

a) A un grupo minoritario de estudiantes les pareció invasivo el tener a un docente como amigo de Facebook puesto que, por un lado sentían que su privacidad estaba siendo expuesta, especialmente para aquellos que tienen conductas que pudieran ser reprochables ante los ojos de un maestro y el tiempo que les iba a significar invertir en esto en desmedro de otras actividades. Después, comprendieron que era de participación libre y que sólo había sido creado para favorecer su aprendizaje y desempeño futuro. Para el resto de estudiantes, no les significó mayor problema, argumentaron que las redes sociales son de uso público y, además, era una forma de aprender de manera más interactiva, dinámica, rápida, fácil y accesible que otras plataformas. Incluso varios estudiantes propusieron que debería ser replicado en otras asignaturas.

b) El muro se saturaba rápidamente. Independiente de que es una desventaja en la cual hay que trabajar y mejorar, los estudiantes mencionaron que también habían foros en tiempo real donde podían opinar de manera instantánea para que así no se generara la disyuntiva de que los docentes creyeran que estaban copiando las respuestas anteriores. Así también, mencionaron que la mayor ventaja es que el chat quedaba visible en el muro, lo que les permitía revisarlo en otro momento, repasar la materia y que las respuestas o dudas generadas por los compañeros también les servían para aprender.

c) No todos tenían la aplicación de Facebook en su teléfono celular, lo que resultó en un gran inconveniente. Sin embargo, ciertos estudiantes hicieron alusión de que todos tenían Facebook y que cada uno manifestaba el interés a lo que realmente quería y que era sólo una excusa.

d) No todos tienen internet en el celular, lo que constituía una excusa, ya que en la Facultad y Universidad hay internet abierto para los estudiantes.

\section{Sugerencias para mejorar el foro virtual por medio de Facebook}

Entre las sugerencias que más destacaron tenemos:

- Aumentar el número de ACC por semana.

- Que fueran tanto ACC de la materia que se vio durante la semana y otros con toda la materia.

- Avisar el horario en que se subirían los ACC a la plataforma para estar preparados.

- Que se vayan desprendiendo más preguntas de los ACC que generaran más debate.

- Subir más videos que complementen los contenidos.

Otras sugerencias que no se enmarcaba dentro de los objetivos del foro: chat personalizados con interacción estudiante-docente de manera privada, lo cual no es el propósito de un foro donde todos opinan, es ahí donde está la riqueza, cuando otro estudiante aprende tanto con sus pares como con el docente que los está retroalimentando. Es en la interacción donde se logra el aprendizaje del grupo, de lo contrario se transformaría en clases personalizadas ${ }^{9}$.

Se realizó triangulación de datos por género, participación en el foro y rendimiento académico en la asignatura. Para la primera, no se observaron diferencias significativas al momento de mencionar las ventajas, desventajas y sugerencias del foro, las opiniones emitidas fueron bastante similares para ambos sexos, sin embargo, el sexo femenino fue mucho más preciso, detallista y certero al momento de hacerlo, lo que concuerda con otras investigaciones similares ${ }^{5}$.

Para la triangulación por participación en el foro, para las sugerencias, la opinión del grupo con alta participación fueron más precisos y asertivos, a diferencia de los de baja participación, los cuales pidieron incorporar ciertas cosas que ya se habían realizado y no se dieron cuenta que ya estaban. Por ejemplo: Se pidió más de un ACC por semana y eran dos a tres por semana, se sugirió que fueran patologías diversas y eran patologías diferentes en cada uno. Por otra parte, este mismo grupo, se escudó bastante en el hecho de que los ACC se saturaban pronto y era la causa de su baja participación siempre.

Para la triangulación por rendimiento académico, para ambos grupos (alto y regular/bajo rendimiento) las ventajas del foro por medio de Facebook superó a cualquier otra experiencia que hayan tenido de este mismo tipo, por lo que las opiniones emitidas fueron 
muy similares, teniendo el mismo valor y significancia para todos. Por el contrario, para las desventajas, los de rendimiento regular/bajo insistieron en excusarse de que no podían participar porque se saturaba rápidamente el foro, además de encontrarlo invasivo y que tenían que invertir más tiempo en desmedro de otras actividades. Es importante hacer notar la defensa que realizaron los estudiantes con alta participación y alto rendimiento, durante el grupo focal, al recomendar a sus compañeros que igual se podía participar, pero sin leer las respuestas antes. Incluso, un estudiante que no podía participar en el foro en vivo, porque tenía otra clase en el mismo horario, manifestó que lo llegaba a revisar después y respondía las preguntas en su cuaderno sin leer las respuestas que estaban en el foro, para hacer el ejercicio de desarrollarlo y después revisaba sus respuestas con las que habían en el muro, eso le había ayudado a aprender más.

\section{Discusión}

El foro virtual mediante la plataforma de Facebook resultó ser un acierto para fomentar las habilidades actitudinales, cognitivas, autorreguladoras y metacognitivas en los estudiantes de Odontología de la UDEC. El análisis cuantitativo evidencia una gran adherencia en la opción -bastante- para la gran mayoría de las preguntas y en el análisis cualitativo pone de manifiesto múltiples y variadas ventajas que ofrece la plataforma en comparación a otras utilizadas por los estudiantes ${ }^{3,12}$. Al respecto, existen innumerables artículos que demuestran que la tecnología ofrece ilimitadas cualidades para el proceso de enseñanzaaprendizaje, donde no sólo se puede interactuar con el estudiante, sino que, además, favorece la ejemplificación de las materias al adjuntar videos o imágenes que complementan los conocimientos de manera instantánea y práctica ${ }^{18}$. Por lo que se podría decir que, la herramienta tecnológica aunada de una red social ofrece mucho más beneficios que el espacio virtual en sí mismo, ya que es mucho más masivo, instantáneo y da la posibilidad de formar grupos o encuentros con base en temas de interés común ${ }^{14,16}$.

Dentro de las variables analizadas, resalta la habilidad actitudinal como una de las que menos adherencia tuvo entre los estudiantes, que se traduce en la conducta y disposición hacia el aprendizaje. Ello podría estar dado porque los estudiantes están acostumbrados a mantenerse a la espera de instrucciones por parte del docente, lo que limita el desarrollo de la autogestión y autorregulación $\mathrm{n}^{6,15}$. Por lo mismo, surge la necesidad de instaurar más metodologías como la presentada, con el propósito de motivar y guiar al estudiante hacia la búsqueda incesante de nuevo conocimiento.

Por otro lado, en la triangulación de datos por género, el sexo femenino manifestó mayor adherencia a las opciones positivas que el sexo masculino, además de ser más detallistas, certeras y precisas en las opiniones dadas en el focus group. Sólo una pregunta resultó ser la excepción - Siento que con el foro he sido estimulado a ser vigilante en las oportunidades de usar el pensamiento crítico-, la cual tuvo mayor adherencia en el género masculino, lo que podría indicar que la tecnología incorporada en la asignatura estimuló más a los varones que a las mujeres. Aunque faltan más elementos de análisis para llegar a una conclusión más certera al respecto.

El análisis cuantitativo se condice con el cualitativo, puesto que los estudiantes que tuvieron más participación en el foro fueron los que lograron mejor rendimiento en la asignatura y, a su vez, manifestaron más ventajas al momento de evaluar la plataforma de Facebook. En cambio, los de baja participación en el foro lograron un menor rendimiento en la asignatura y son los que más desventajas mencionaron. Es así que Rosário, et al. ${ }^{19}$ refieren que "Los alumnos que autorregulan su aprendizaje son proactivos en cuanto a sus esfuerzos por aprender, ya que son conscientes de sus habilidades y limitaciones y, además, su comportamiento de estudio está guiado por objetivos y estrategias que los ayudan a alcanzarlos".

Por lo mismo, los alumnos de baja participación y regular/bajo rendimiento se excusaban bastante en diversos motivos que les impedía participar, como que el foro se saturaba muy rápido con las respuestas de otros compañeros y ya no respondían porque el docente pensaría que ellos estaban copiando las respuestas, el sentirse invadidos al tener un docente que vería lo que publicaban en Facebook y el tiempo que les iba a significar invertir en desmedro de otras actividades. Ello demuestra que las causas y circunstancias que le impidieron participar de manera activa en el foro siempre se debieron a causas externas, en ningún momento hicieron un juicio introspectivo de las causas, evidenciando una escasa reflexividad que limita el desarrollo de la autorregulación y metacognición ${ }^{20}$.

Ahora bien, todo lo anterior demanda un cambio de paradigma, tanto en los docentes como en los mismos estudiantes, puesto que los primeros están acostumbrados a utilizar las plataformas institucionales para generar espacios de aprendizaje, siendo que ya 
hemos visto que a los estudiantes no les agradan por encontrarlos poco atractivos y limitantes ${ }^{7,10,11}$. Por otro lado, a algunos alumnos no les gustó que les invadieran su espacio en las redes sociales, aunque es sabido que son espacios abiertos. Igualmente, es necesario recordar que los estudiantes aceptaron voluntariamente en participar, por lo que a ninguno se le obligó a ser parte del grupo cerrado de Facebook.

\section{Conclusiones}

El mundo cambia y avanza sigilosamente, pretender mantener un estilo o forma metodológica que asegure un buen aprendizaje a lo largo del tiempo es imposible. Lo importante es saber adaptarse a los cambios, conocer las nuevas herramientas disponibles y tener el respaldo necesario para diseñar y planificar estrategias que aseguren el logro de los objetivos que se desean alcanzar en el estudiante ${ }^{9,12,15,18}$.

Resulta trascendental poder innovar y utilizar las nuevas herramientas tecnológicas que motiven y estimulen la curiosidad de los estudiantes, para fomentar la actitud, cognición, autorregulación y metacognición, para desarrollar en ellos la incesante necesidad de búsqueda de conocimiento, que les permitirá enfrentarse a un mundo cada vez más acelerado, cambiante y globalizado. Tal como Ruiz-Corbella, et al. ${ }^{9}$ refieren "debemos aprender para mejorar el diseño de nuestros cursos, ya sea introduciendo en los foros la atención a las respuestas, necesidades e intereses que los estudiantes buscan en estas redes, o bien contando, de forma paralela, con otros espacios de interacción con ellos, o incluso valorando la posibilidad de integrar apps de redes sociales en nuestros entornos virtuales, como reclamo de una docencia tendiente al 2.0".

La presente investigación no está exenta de ciertas limitaciones, dado que la muestra es pequeña y sólo involucra a una carrera en específico. Sin embargo, pretende ser el impulso inicial para que otras universidades y carreras incorporen las redes sociales en el proceso de enseñanza-aprendizaje y compartan las investigaciones desarrolladas al respecto.

\section{Conflicto de intereses}

El presente trabajo ha sido realizado sin ningún fin lucrativo, además los investigadores declaran no tener ningún conflicto de interés que pudiera influir en los resultados de este estudio.

\section{Referencias}

1. Castro S, Guzmán B, Casado D. Las TIC en los procesos de enseñanza y aprendizaje. Laurus. 2007; 13(23): 213-234.

2. Jaramillo C, Chávez J. TIC y educación en Chile: una revisión sistemática de la literatura. Nuev Ideas Informat Edu TISE. 2015; 221-231.

3. Torres Gastelú CA, Moreno Coatzozón G. Inclusión de las TIC en los escenarios de aprendizaje universitario. Apertura. 2013; 5(1): 48-65.

4. Chávez López J, del Toro Valencia M, López O. Blog, correo electrónico y foros temáticos: su uso, dominio y actitud en estudiantes de educación medio superior de México. Hamut'ay. 2017; 4(2): 45-54. doi: 10.21503/hamu.v4i2.1471.

5. Castro-Romero O. Uso de los medios sociales como herramienta de aprendizaje en educación superior: análisis comparativo entre México y Corea del Sur. Sinéctica. 2015; 44: 1-16.

6. Torres Gastelú C, Moreno Coatzozón G. Inclusión de las TIC en los escenarios de aprendizaje universitario. Apertura. 2013; 5(1): 48-65.

7. Chile es el país que más creció en acceso a internet y alcanza el octavo lugar en el mundo en penetración de la red. Emol.com; 2018.

8. Economía y negocios online. Chile es calificado como el mejor país latinoamericano en acceso y uso de internet; 2018.

9. Ruiz-Corbella M, Fernández AD, García-Blanco M. Participación en foros virtuales en cursos masivos (UNED). Redie. 2016; 18(3): 121-134.

10. Nass L, Mendoza M, Millanao L, Ortega R. Evaluación de una plataforma educativa en la Universidad de Concepción, Chile. Educ Med Super. 2017; 31(1): 99-113.

11. Díaz-Barriga A. TIC en el trabajo del aula, impacto en la planeación didáctica. Rev Iberoam Edu Super. 2013; 10(4): 3-21. doi:10.1016/S20072872(13)71921-8.

12. Castro N, Suárez X, Soto V. El uso del foro virtual para desarrollar el aprendizaje autorregulado de los estudiantes universitarios. Innov Educat. 2016; 16(70): 23-41.

13. Herrera AM. Una mirada reflexiva sobre las TIC en Educación Superior. Redie. 2015; 17(1): 1-4.

14. Hermosa del Vasto PM. Influencia de las tecnologías de información y comunicación (TIC) en el proceso enseñanza-aprendizaje: una mejora de las competencias digitales. Rev Cient Gen José María Córdova. 2015; 13(16): 121-132. doi: 10.21830/19006586.34. 
15. Alvarez-Cruces D, Otondo-Briceño M. Transferencia de aprendizajes en estudiantes de Odontología de la Universidad de Concepción, Chile. Educ Med Super. 2018; 32(4).

16. De la Hoz LP, Acevedo D, Torres J. Uso de redes sociales en el proceso de enseñanza y aprendizaje por los estudiantes y profesores de la Universidad Antonio Nariño, sede Cartagena. Form Univ. 2015; 8(4): 77-84. doi: dx.doi.org/10.4067/S071850062015000400009 .

17. Fedorov A. Foro virtual como una estrategia metodológica para el desarrollo del pensamiento crítico en la universidad. Innov Edu. 2008; 21(3): 102-113.
18. González Rodríguez R, Cardentey García J, González García X. Consideraciones acerca del empleo de las tecnologías de la información en la enseñanza universitaria. Educ Med Super. 2015; 29(4): 837-842.

19. Rosário P, Pereira A, Högemann J, Nunes AR, Figueiredo M, Núñez J, et al. Autorregulación del aprendizaje: una revisión sistemática en revistas de la base SciELO. Univ Psychol. 2014; 13(2): 781797. doi:10.11144/Javeriana.UPSY13-2.aars.

20. Arias Gallegos W, Zegarra Valdivia J, Justo Velarde O. Estilos de aprendizaje y metacognición en estudiantes de psicología de Arequipa. Liberabit. 2014; 20(2): 267-279.

\section{ANEXO 1: Encuesta de opinión de Andrei Fedorov.}

\section{Pregunta}

1. Siento que a través del foro mi capacidad de reflexión ha sido incrementada.

2. Estimo que mi motivación por aprender ha sido inspirada por lo vivido en el foro.

3. He notado que el foro me ha facilitado la comprensión de las ideas complejas.

4. He descubierto que mi capacidad de analizar problemas se ha incrementado a través del foro.

5. Siento que con el foro he sido estimulado a ser vigilante en las oportunidades de usar el pensamiento crítico.

6. He percibido que mi claridad de expresión ha mejorado a partir de mi experiencia en el foro.

7. Siento que el espíritu investigativo ha sido impulsado por medio del foro.

8. Siento que el foro fomenta el respeto por la libre opinión de los demás.

9. He descubierto que mi capacidad argumentativa se ha mejorado a consecuencia de mi participación en el foro.

10. He aprendido a valorar los consensos a través del foro.

11. Considero que con el foro he acentuado mi persistencia ante una temática difícil.

12. Creo que una de las consecuencias del foro es que ahora estoy más consciente de repensar mis opiniones antes de expresarlas.

13. Opino que el foro en mi caso ha despertado la curiosidad intelectual.

14. Después de mi experiencia con el foro, pienso que mi sentido crítico es más oportuno.

15. Opino que a partir del foro he aprendido a confrontar distintas ideas.

16. Percibo que mi nivel de honestidad para enfrentar mis propias debilidades se ha consolidado debido a mi participación en el foro.

17. Con la experiencia en el foro mi capacidad de negociación se ha intensificado.

18. Creo que con el foro mi capacidad de generar mi propia opinión ha sido fortalecida.

19. Siento que me he obligado a considerar los factores contextuales como el punto de partida de mis opiniones durante el desarrollo del foro.

20. Opino que la necesidad de mantenerme bien informado se ha fomentado a través del foro.

Fuente: http://www.redalyc.org/html/1794/179420843006/ 


\section{ANEXO 2: Guión temático del Focus Group}

Objetivo: Conocer la percepción de los estudiantes en relación a la utilización de la plataforma de Facebook para un foro virtual en la asignatura Diagnóstico Integrado de la carrera de Odontología de la Universidad de Concepción.

\begin{tabular}{llll}
\hline \multicolumn{1}{c}{ Categoría } & \multicolumn{1}{c}{ Subcategorías } & \multicolumn{1}{c}{ Preguntas claves } \\
\hline & Ventajas & 1. ¿Cuáles son las ventajas de utilizar la plataforma de Facebook para el foro virtual en \\
comparación a otras?
\end{tabular}

